\section{Compreensão da terapia anti-retroviral: uma aplicação de modelo de traço latente}

\author{
Patient's understanding of information on \\ antiretroviral therapy: an application \\ of the latent trait model
}

\author{
1 Faculdade de Medicina, \\ Universidade Federal de \\ Minas Gerais, Belo Horizonte, \\ Brasil. \\ 2 Grupo de Pesquisas em \\ Epidemiologia e Avaliação \\ em Saúde, Universidade \\ Federal de Minas Gerais, Belo \\ Horizonte, Brasil. \\ 3 Faculdade de Farmácia, \\ Universidade Federal de \\ Minas Gerais, Belo Horizonte, \\ Brasil. \\ 4 Instituto de Ciências Exatas, \\ Universidade Federal de \\ Minas Gerais, Belo Horizonte, \\ Brasil. \\ Correspondência \\ F. A. Acurcio \\ Departamento de Farmácia \\ Social, Faculdade de \\ Farmácia, Universidade \\ Federal de Minas Gerais \\ Av. Antônio Carlos 6627, \\ Campus Pampulha, FAFAR, \\ sala 1048B2, Belo Horizonte \\ MG 31270-901, Brasil. \\ acurcio@medicina.ufmg.br
}

\begin{abstract}
The aim of this study was to develop a score to determine the level of understanding regarding information on antiretroviral therapy (ART) among patients initiating treatment. This was a cross-sectional analysis based on interviews with HIV patients in outpatient public referral centers (Belo Horizonte, Minas Gerais State, Brazil). The score for patients' understanding of their medicines was obtained using a latent trait model, estimated by the Item Response Theory, based on the concordance between each patient answer and the written prescription. Hierarchical linear regression was used to assess patients' global understanding of ART, considering each class of drugs (level 1) and the individual (level 2). Among 406 patients, 37.9\% failed to reach a minimum level of understanding of their treatment. The item with the highest level of difficulty was "precaution in use". The item "dosage" showed the most varied understanding of ART. A high proportion of patients displayed minimal understanding of ART, indicating a high potential risk for non-adherence to therapy. It is thus necessary to identify factors associated with insufficient understanding of ART.
\end{abstract}

HIV; Anti-Retroviral Agents; Drugs with Prescription

\author{
Maria das Graças B. Ceccato 1,2 \\ Francisco A. Acurcio 1,2,3 \\ Cibele C. César 2,4 \\ Palmira F. Bonolo 2 \\ Mark D. C. Guimarães 1,2
}

\section{Introdução}

No Brasil, uma das medidas fundamentais para o controle da epidemia de HIV/AIDS tem sido a garantia de acesso aos medicamentos anti-retrovirais (ARV) pelos indivíduos que deles necessitem. No entanto, a efetividade da terapia ARV requer um alto nível de adesão ao tratamento (95\%$100 \%)^{1}$. A compreensão insuficiente sobre o uso correto dos medicamentos e a falta de informação sobre os riscos advindos do não cumprimento da terapia prescrita são aspectos que podem levar o individuo a não aderir ao tratamento 2 . Por isso, fornecer orientação e informação aos pacientes sobre os seus medicamentos é um princípio essencial da farmacoterapia racional 3 que busca assegurar sua adequada utilização.

Existem na literatura poucas pesquisas enfocando aspectos relativos à informação obtida pelos pacientes sobre os medicamentos prescritos e sua percepção dessas informações, sendo ainda mais raras aquelas referentes à terapia ARV. Embora haja consenso de que se deva medir o grau de compreensão da prescrição médica pelo paciente, não há plena concordância sobre o que constitui este conhecimento do regime terapêutico nem quanto à forma de medi-lo. Múltiplas estratégias operacionais e várias características do tratamento têm sido utilizadas para a investigação e mensuração desta compreensão em diferentes combinações. São aplicados questionários ou testes de conhecimento enfo- 
cando um conjunto de itens, tais como nome do medicamento, indicação, dose, freqüência de administração, duração do tratamento, efeitos adversos, precauções e instruções especiais sobre administração, e o reconhecimento do medicamento em presença de outros medicamentos $4,5,6,7,8,9,10$.

Uma das estratégias utilizadas para aferir o grau de compreensão do paciente sobre o regime terapêutico é o cálculo de escores, a partir da atribuição de pontuação às respostas corretas de um teste de conhecimento, segundo sua relevância, com determinação de pontos de corte para classificação dos escores. Observa-se uma grande diversidade quanto ao ponto de corte e níveis de classificação da compreensão obtida 5,6. A variabilidade de tais medidas de compreensão pode dificultar a identificação de aspectos que contribuem para equívocos de interpretação pelo paciente, como também comprometer a comparabilidade entre os resultados de investigações 4 . Ademais, alguns estudos com o mesmo enfoque utilizaram metodologias semelhantes e obtiveram resultados bem diferentes entre si 8,10. Assim, o desenvolvimento de uma medida acurada da compreensão, por parte dos pacientes, dos esquemas terapêuticos utilizados adquire grande relevância para subsidiar ações dos profissionais de saúde no sentido de garantir uma maior efetividade no tratamento, especialmente no início da terapia.

O modelo da Teoria de Resposta ao Item (TRI) 11 mostra-se uma alternativa adequada para esta mensuração, ao evitar a simples agregação de diferentes indicadores do nível de compreensão do tratamento. O propósito da TRI é estimar uma determinada característica que não pode ser medida diretamente, denominada traço latente. Neste caso, a compreensão do tratamento é o construto ou traço latente que se deseja medir e que se manifesta por meio de variáveis relativas ao tratamento (i.e. nome do medicamento, dose, precauções, freqüência de administração, reações adversas e alimentação) 12,13. A TRI destaca-se por ser um método não arbitrário, que considera cada item particularmente. Entre as vantagens do modelo está o fato de permitir a estimação do construto mesmo para aqueles indivíduos que não disponham de informação sobre todos os itens investigados, pois trata de forma eficiente os dados ausentes. Uma outra vantagem é propiciar a comparabilidade dos resultados obtidos para grupos de indivíduos diferentes e testes diferentes, desde que esses testes tenham alguns itens comuns que meçam um mesmo traço latente. Isso porque, a estimação do escore total depende dos itens que compõem o teste, e não do grupo de respondentes 14,15.
Este trabalho integra o Projeto Adesão ao Tratamento Anti-retroviral (ATAR), um estudo prospectivo concorrente delineado para verificar a incidência e os determinantes da não-adesão ao tratamento ARV em indivíduos infectados pelo HIV/AIDS 16. Em um trabalho anterior 17 avaliouse a compreensão de informações relativas ao tratamento ARV. O nível de compreensão foi então medido por meio de um escore, arbitrandose diferentes pontos para cada item enfocado, de acordo com a sua importância para o uso seguro dos medicamentos. Entretanto, a adoção desta estratégia de avaliação apresenta como principal limitação o fato de não considerar os diversos graus de dificuldade observados para cada item no processo de assimilação e compreensão do tratamento, segundo as características do paciente e as condições de uso do medicamento. Com o intuito de contribuir para a discussão e o refinamento metodológico de como mensurar a compreensão do tratamento, o presente estudo teve por objetivos desenvolver um novo escore da compreensão do paciente para cada medicamento prescrito, utilizando um modelo de traço latente estimado pela TRI e determinar o nível global de compreensão de informações sobre a terapia ARV em indivíduos no início do tratamento.

\section{Material e métodos}

O Projeto ATAR 16 foi conduzido em dois serviços públicos de saúde de referência para a assistência ambulatorial especializada em HIV/AIDS, em Belo Horizonte, Minas Gerais, Brasil, atendidos no período de 2001 a 2003.Os critérios de elegibilidade incluíram todos os indivíduos com evidência laboratorial de infecção pelo HIV e/ ou diagnóstico de AIDS, com idade igual ou superior a 18 anos, sendo 16 anos para mulheres grávidas, admitidos para sua primeira prescrição de ARV. Aqueles que aceitaram voluntariamente participar do estudo assinaram o consentimento informado esclarecido. Foram excluídos os indivíduos que não apresentaram condições mínimas de autonomia para decidir acerca da adesão ao tratamento, bem como aqueles indivíduos em uso de medicamentos devido a acidente de trabalho. O projeto foi aprovado pelas instituições onde a pesquisa foi desenvolvida e pelo Comitê de Ética em Pesquisa (COEP) da Universidade Federal de Minas Gerais (UFMG; no. 106/99), estando de acordo com todos os princípios éticos e legislações vigentes de pesquisas que envolvem seres humanos. 


\section{Nível de compreensão}

Os dados foram obtidos por meio de entrevistas confidenciais estruturadas realizadas na entrevista basal do estudo prospectivo, logo após a primeira dispensação dos medicamentos ARV. Os dados clínicos foram obtidos nos prontuários médicos, imediatamente antes da primeira prescrição de ARV. Para os dados relativos aos medicamentos prescritos, a receita médica foi transcrita durante a entrevista. Para alguns pacientes que não portavam a receita no momento da entrevista, foi possível recuperar as informações de prescrição posteriormente. Permitiu-se ao paciente consultar a receita, ou embalagem do medicamento ou qualquer anotação que ele tivesse em mãos, para responder sobre sua compreensão quanto à terapia instituída. O nível de compreensão da prescrição de anti-retrovirais foi avaliado a partir de seis itens: nome do medicamento, dose, precauções, freqüência de administração, reações adversas e alimentação. Para as informações relativas a reações adversas, precauções e recomendações quanto à alimentação, considerou-se a possível ocorrência de orientação verbal e, quando a informação não constava na receita comparou-se a resposta do paciente com as informações existentes no Consenso Brasileiro 2. A interpretação da concordância entre as respostas do paciente e a informação na prescrição foi feita por dois revisores. Foram consideradas respostas dicotômicas (certa/errada). Para avaliar a resposta do paciente como correta exigiu-se a concordância entre os dois revisores. Em caso de discordância, um terceiro revisor foi consultado.

Teoria de Resposta ao Item e a medida dos construtos explicativos

A partir da avaliação de concordância entre as respostas do paciente e a informação na prescrição, o estudo percorreu as seguintes etapas: (i) análise exploratória dos dados; (ii) estimativa da compreensão de cada item selecionado; (iii) estimativa da compreensão de cada ARV; (iv) estimativa da compreensão global da terapia; e, (v) construção e interpretação da escala dessa compreensão global.

\section{Análise exploratória dos dados}

Realizada por meio do cálculo da proporção de acertos de cada item e do coeficiente de correlação bisserial. Este coeficiente mede a correlação do resultado de um item em particular do teste com o total de acertos e fornece um diagnóstico preliminar, sendo útil para a escolha daqueles itens que de fato apresentam consistência interna e se associam bem ao escore que será produzido 12,18 .

\section{Estimativa da compreensão de cada item}

Para desenvolver este novo escore de compreensão por meio de modelo da TRI, considerouse que cada item selecionado é um indicador da compreensão da terapia ARV, característica latente e não observável do paciente. A relação entre a resposta obtida e o nível de compreensão é observada na curva característica do item (CCI), que descreve a relação entre a compreensão $(\theta)$ dos respondentes e a probabilidade de acerto, sendo função dos parâmetros do item. Os modelos da TRI variam com o tipo de item utilizado 13,15 . Neste estudo, ajustou-se para cada um dos seis itens selecionados, o modelo logístico unidimensional de dois parâmetros de Birnbaum 19,20 - discriminação $\left(a_{i}\right)$ e a dificuldade $\left(b_{i}\right)$ - que é apropriado para itens com respostas dicotômicas e modela a probabilidade de um indivíduo responder corretamente a uma determinada questão, dado o seu conhecimento sobre o assunto. O índice de discriminação $\left(a_{i}\right)$ indica o quanto um item é capaz de diferenciar indivíduos com base no conhecimento de determinado assunto, neste caso a compreensão sobre a terapia ARV. Em geral, assume-se que os itens cujos parâmetros $a$ apresentaram valores inferiores a 0,30 representam uma baixa discriminação 12,18,21. O índice de dificuldade $\left(b_{i}\right)$ está relacionado com a probabilidade de acerto ao item e é medido na mesma escala da compreensão $(\theta)$, o que permite estabelecer quais itens o indivíduo terá mais chance de acertar 12,15. Um melhor detalhamento dos fundamentos teóricos e matemáticos da TRI podem ser obtidos na literatura específica $15,20,21,22,23,24,25$.

\section{Estimativa da compreensão de cada ARV}

Por meio da análise do modelo TRI, as CCIs foram determinadas e os parâmetros dos itens estimados pelo Método de Máxima Verossimilhança. Os valores dos escores $(\theta)$ da compreensão do paciente para cada medicamento ARV foram então estimados por meio de método bayesiano.

\section{Estimativa da compreensão global da terapia}

Para analisar a compreensão global, enfocando todos os ARV utilizados pelo paciente, realizouse uma regressão linear multinível 26. Ao se considerar o medicamento como unidade de nível 1 e o paciente como unidade de nível 2, foi possível estimar o escore global de compreensão da tera- 
pia pelo intercepto do modelo nulo (ANOVA com fator aleatório), levando-se em conta a correlação entre as observações, sem perder informações devido ao desbalanceamento da amostra (número diferente de medicamentos por paciente).

\section{Construção e interpretação da escala de compreensão global da terapia ARV}

Os parâmetros dos itens foram, inicialmente, estimados na escala $(0 ; 1)$, em que a média é 0 e o desvio-padrão é 1. Para facilitar a utilização e o entendimento da escala de compreensão, realizou-se transformação linear em todos os valores originais obtendo-se uma nova escala contínua com média igual a 50 e desvio-padrão igual a 515 .

O próximo passo foi a identificação de itens âncora que permitem a interpretação da escala em certos pontos pré-fixados - os níveis âncora. Para que um item seja âncora em determinado nível, deve satisfazer a certas condições matemáticas, ou seja, em qualquer ponto da escala da compreensão é acertado por pelo menos $65 \%$ de pacientes com este grau de desempenho. Ao mesmo tempo, são itens respondidos corretamente por uma minoria de pacientes (35\%) situados no ponto imediatamente inferior e pela quase totalidade (95\%) situados no ponto imediatamente superior 15. Assim, foram obtidos seis níveis âncora (um para cada item investigado sobre os ARV). As descrições dos itens são progressivas e cumulativas, ou seja, os conhecimentos descritos em um nível inferior estão contidos no superior. Deste modo, a escala nos permite identificar quais são os itens relativos ao conhecimento dos ARV que os pacientes dominam ou não. Finalmente, considerando-se a literatura 6,17 e o uso racional de medicamentos 3 , definiu-se um ponto de corte nessa escala que estabelece um nível mínimo satisfatório de compreensão global da terapia ARV. Assim, indivíduos que apresentaram conhecimento insuficiente para responder corretamente a 3 itens - o nome do ARV, a freqüência de administração e a dose prescrita -, não alcançaram esse ponto de corte e, portanto, não possuem o nível de compreensão necessário para utilizar os ARV de maneira segura e adequada.

Os dados foram digitados em Paradox (Borland International, Scotts Valley, Estados Unidos) e analisados em SAS (SAS Inst., Cary, Estados Unidos), BILOG-MG 3.0 (Scientific Software International, Lincolnwood, Estados Unidos) para estimativas dos parâmetros e MLWin 2.0 (Centre for Multilevel Modelling, Bristol, Reino Unido) para análise multinível.

\section{Resultados}

As características avaliadas dos 406 participantes do estudo mostram predominância da população abaixo de 35 anos (53,7\%; mediana $=35,0)$, do sexo masculino (55,9\%), afrodescendentes $(77,2 \%)$, solteiros $(60,6 \%)$ com escolaridade inferior a 8 anos $(52,7 \%$; mediana $=7,0)$. Observou-se uma grande maioria com renda igual ou inferior a $\mathrm{R} \$ 360,00$, equivalente a 1,2 salários mínimo $(71,5 \%$; mediana $=360,00)$ e sem plano de saúde (77,3\%).

Em relação à terapia $\mathrm{ARV}$, foram utilizados vinte e dois regimes de ARV distintos. Esquemas de monoterapia com zidovudina ou duplos foram utilizados por 30 pacientes $(7,4 \%)$, sendo que a quase totalidade $(\mathrm{n}=29)$ era destinada à profilaxia para gestantes; $355(87,4 \%)$ eram esquemas tríplices e $21(5,2 \%)$ eram esquemas quádruplos. Os regimes mais freqüentes compreenderam associações com zidovudina (AZT), lamivudina (3TC) ou efavirenz (EFZ), sendo os principais esquemas prescritos inicialmente as combinações AZT+3TC+nelfinavir (NFV); $\mathrm{AZT}+3 \mathrm{TC}+\mathrm{EFZ}$; e AZT+3TC+nevirapina (NVP), para, respectivamente $35 \%, 29,1 \%$, e $16,7 \%$ dos participantes. A utilização concomitante de fármacos de uso contínuo e regular aos ARV foi relatada por 157 (40,6\%) participantes. O número de comprimidos diários do esquema ARV variou entre 1 a 21, com média de 8,4 $\pm 4,3$ comprimidos (mediana $=8,0)$. Quase a metade dos participantes era sintomática ou possuía condições clínicas definidoras para a AIDS (49,6\%) 27 e 90,7\% apresentavam contagem de células TCD4+ inferior a 500 células $/ \mathrm{mm}^{3}$.

\section{Interpretação dos parâmetros dos itens} utilizados no desenvolvimento do escore

Foram prescritos 872 ARV para os 406 participantes do estudo. A análise da concordância indicou um índice de acerto $92,4 \%$ para a dose a ser tomada, de $83,3 \%$ para freqüência de administração e de $72,2 \%$ para o nome do medicamento. A proporção de acerto foi muito baixa para o item precauções (19,7\%) (Tabela 1). Já o coeficiente de correlação bisserial variou entre 0,09 e 0,44 tendo o item precauções obtido o menor coeficiente $(0,09)$, o que indica baixa correlação entre acertos dessa questão com o escore total do teste. Apesar disto, esse item não foi excluído das análises posteriores devido ao caráter exploratório desta avaliação.

A investigação da qualidade de cada um dos itens avaliados, mostrou neste estudo, que o parâmetro de discriminação $a$ variou entre 0,30 e 1,29. Dentre os itens avaliados, apenas um 
Estimativa dos parâmetros dos itens relacionados à compreensão do paciente sobre os medicamentos prescritos $(n=872)$. Belo Horizonte, Minas Gerais, Brasil, 2001-2003.

\begin{tabular}{lcccc}
\hline Itens & $\begin{array}{c}\text { Proporção de } \\
\text { acertos (\%) }\end{array}$ & $\begin{array}{c}\text { Correlação } \\
\text { bisserial }\end{array}$ & $\begin{array}{c}\text { Parâmetro de } \\
\text { dificuldade * }\end{array}$ & $\begin{array}{c}\text { Parâmetro de } \\
\text { discriminação * }\end{array}$ \\
\hline Dose & 92,4 & 0,24 & $-1,89(0,18)$ & $1,29(0,28)$ \\
Freqüência de administração & 83,3 & 0,14 & $-1,82(0,24)$ & $0,52(0,08)$ \\
Nome ARV & 72,2 & 0,22 & $-1,54(0,23)$ & $0,43(0,07)$ \\
Alimentação & 69,0 & 0,44 & $-1,27(0,20)$ & $0,45(0,08)$ \\
Reações adversas & 58,8 & 0,13 & $-0,78(0,19)$ & $0,30(0,05)$ \\
Precauções & 19,7 & 0,09 & $2,54(0,47)$ & $0,35(0,07)$ \\
\hline
\end{tabular}

* Estimativa (erro padrão).

(reações adversas) apresentou um valor limítrofe enquanto todos os demais superaram o valor crítico (Tabela 1). Os itens reações adversas e precauções apresentaram baixa discriminação $(0,21$ 0,37), os itens nome, alimentação e freqüência de administração discriminação moderada $(0,38$ a 0,78$)$, e o item dose discriminação muito alta $(>1,00) 21$. Os parâmetros de dificuldade $b$, que tipicamente, variam entre -3 (itens extremamente fáceis) a 3 (itens extremamente difíceis) na escala utilizada 18,21, indicam que os itens dose, freqüência de administração, alimentação, nome e reações adversas são considerados fáceis enquanto que o item precaução foi considerado muito difícil $(b=2,54)$.

As CCIs, apresentadas na Figura 1, propiciam melhor visualização e complementam a análise dos dados obtidos. Quanto maior o poder de discriminação do item sobre a compreensão, mais elevado o valor de $a$ e mais acentuada a inclinação da CCI. Observa-se que o item dose $(a=1,29)$ apresenta a CCI com inclinação mais acentuada. Nos itens com discriminação elevada, variações de probabilidade de acerto estão mais fortemente associadas à compreensão. Assim, o item dose foi o que mais diferenciou os pacientes quanto à compreensão da terapia ARV.

\section{Interpretação das escalas da compreensão} global da terapia ARV

O nível de compreensão da prescrição da terapia ARV, estimado por meio de modelo hierárquico de 2 níveis, na escala $(50 ; 5)$, apresentou média de 49,93 e amplitude entre 32,57 e 57,73 . Os pontos selecionados da escala, em ordem crescente correspondem aos níveis âncora (Figura 2). Este procedimento possibilitou diferenciar os indivíduos, seqüencialmente, naqueles que não possu- íam conhecimento mínimo para acertar nenhum item do esquema ARV prescrito, ou naqueles que acertaram apenas dose, ou dose mais freqüência e assim sucessivamente. Observou-se que a proporção de indivíduos que não atingiram um nível mínimo de compreensão do tratamento, ou seja, não tiveram conhecimento suficiente para acertar os itens dose, freqüência de administração e nome do ARV (49,6 pontos na escala), foi de $37,9 \%$.

\section{Discussão}

O principal objetivo deste estudo foi estimar escores do construto compreensão da terapia ARV por meio da TRI, para uma mensuração mais acurada de seu nível, dada a influência que essa compreensão pode ter na efetividade no tratamento. Encontrou-se uma proporção de 37,9\% de indivíduos que não atingiram um nível mínimo de compreensão da terapia, o que sinaliza um alto risco potencial de não adesão ao tratamento. Essa proporção foi maior do que a encontrada (26,3\%) em trabalho anterior 17 estudando a mesma população. A diferença observada pode ser devida, principalmente, a um aumento de sensibilidade proporcionado pelo método de mensuração empregado no estudo, o que reforça a adequação de sua escolha. Um outro resultado relevante obtido com esse método foi a comprovação das distintivas características dos itens avaliados (e.g. coeficiente de correlação, índice de discriminação, índice de dificuldade), indicando a necessidade de revisão das estratégias freqüentemente utilizadas para aferir o grau de compreensão do paciente baseadas no cálculo de escores. Chama-se atenção para uma fragilidade dessas estratégias, que em geral 
Figura 1

Curvas características dos itens (CCI). Belo Horizonte, Minas Gerais, Brasil, 2001-2003.
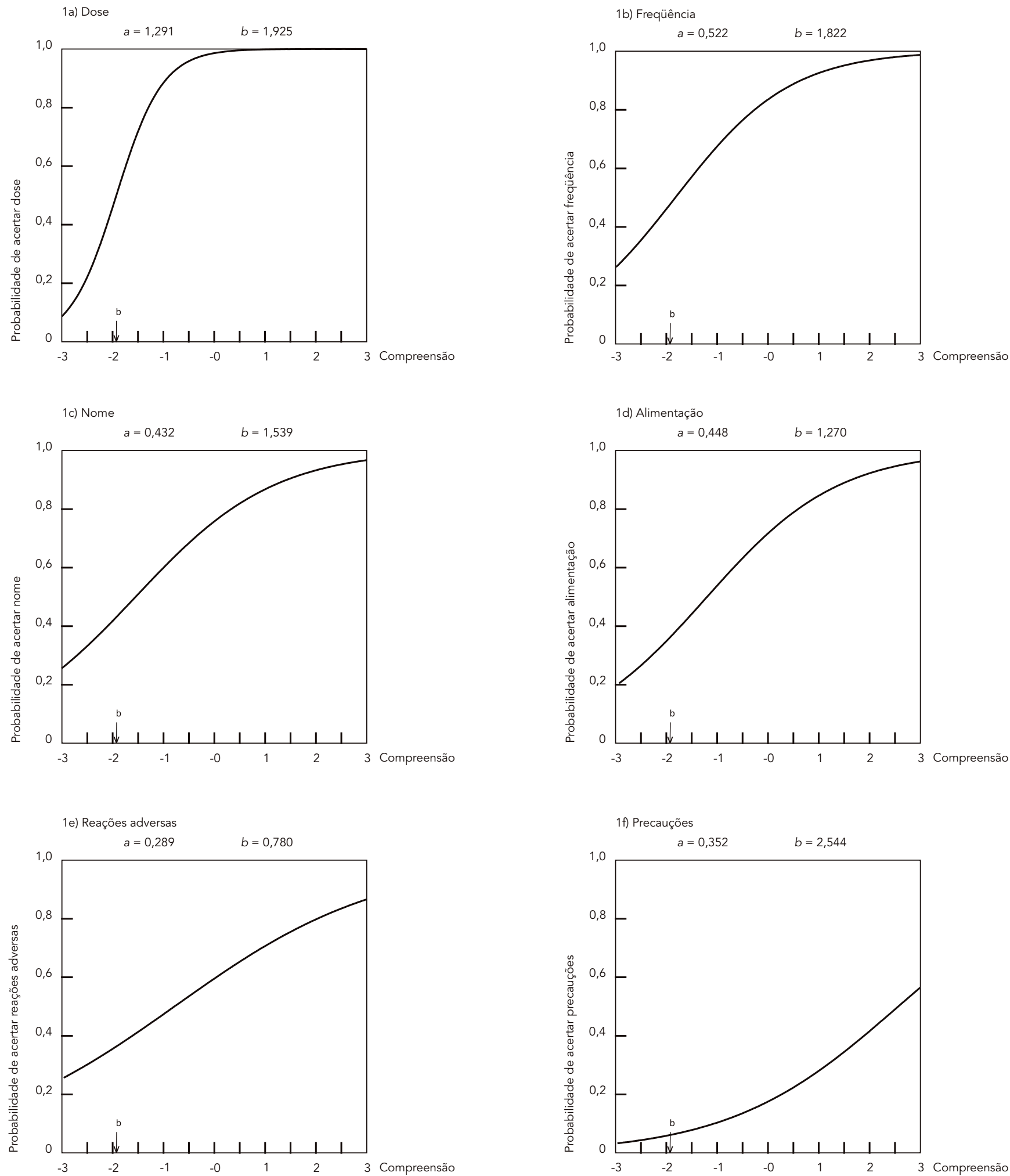


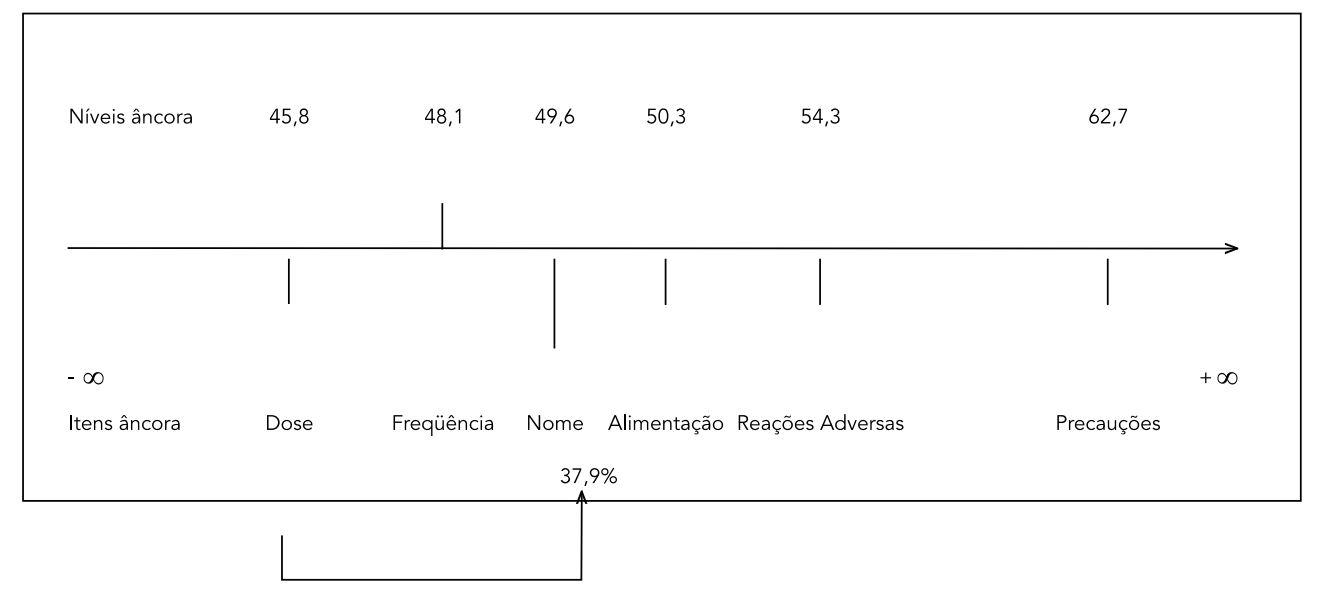

atribuem o mesmo peso para itens heterogêneos quanto à complexidade ou arbitram diferentes valores para cada item, sem considerar o grau de dificuldade na composição do escore final. Ascione et al. ${ }^{4}$ demonstraram que o conceito "conhecimento sobre o medicamento" abrange diferentes itens que não são equivalentes como indicadores na representação deste conceito. Assim, a importância de cada item analisado deve ser distinguida de acordo com as características do paciente, do tratamento e das condições em que o medicamento será utilizado 6 . Os resultados do presente estudo corroboram a conclusão destes autores, pois evidenciam as características distintas de cada item analisado pela TRI, segundo seus parâmetros. A estimativa dos níveis de compreensão da prescrição médica mostrou-se dependente dos diferentes graus de conhecimento do paciente para acertar os itens avaliados para cada ARV.

A compreensão do paciente quanto à terapia ARV prescrita foi definida com base na literatura disponível, selecionando itens indicadores do construto compreensão da terapia medicamentosa, em estudos com o mesmo enfoque $4,5,6,7,8,9,10$. A escala desenvolvida é caracterizada pelos itens selecionados, logo, a qualidade da escala depende da qualidade desses itens, que devem ter índices de dificuldade e de discriminação aceitáveis e correlacionados com o conjunto de questões aplicadas. A quantidade de itens envolvidos deve ser suficiente tanto para caracterizar bem cada ponto da escala, quanto para possibilitar que a escala possa ter vários níveis. Uma boa escala de compreensão será conseqüência da aplicação de um número razoável de itens de boa qualidade (com altos níveis de discriminação e diferentes níveis de dificuldade), e um número suficiente de indivíduos com os mais variados níveis de compreensão. Os problemas que poderiam ocorrer com os parâmetros dos itens correspondem a um valor do índice de discriminação $a$ abaixo do valor crítico 0,30 ; um índice de dificuldade $b$ acima do valor crítico 2,95 ou abaixo de -2,95 ou, ainda, valor negativo da correlação bisserial 18 . No presente trabalho, os itens selecionados apresentaram essas propriedades dentro de padrões aceitáveis 28 . Entretanto, considerando conjuntamente os valores obtidos pelos dois índices, observa-se que os itens estudados conseguem discriminar em níveis baixos de habilidade. O único item que apresentou dificuldade alta e que, potencialmente, poderia discriminar pacientes com alto nível de compreensão, apresentou índice de discriminação muito baixo, próximo ao critico.

As análises indicaram que os itens se agruparam coerentemente, formando uma escala precisa. Observou-se que o item precauções apresenta dificuldade muito elevada $(b=2,54)$ e baixa discriminação $(a=0,35)$. Observou-se também baixo índice de correlação bisserial, o que indica que não há boa correlação entre acertos e erros desse item com o escore total do teste, não se associando bem ao escore produzido. Em relação ao item reações adversas, observou-se um valor 
limítrofe do parâmetro $a(a=0,30)$. A dificuldade mais elevada para o item precauções e uma menor facilidade de acerto para reações adversas e alimentação mostra coerência com os resultados de outras análises que incluíram esses itens $6,19,29,30$. Uma proporção muito pequena dos indivíduos respondeu a essas questões e uma proporção menor ainda os acertou. A falta ou insuficiência de informação fornecida diretamente ao paciente e nas receitas médicas pode ser devida a uma valoração secundária destes itens. Ademais, a probabilidade do paciente, por auto-sugestão, manifestar reações adversas informadas pelos profissionais de saúde, pode desestimular um esclarecimento detalhado sobre este item, pelas possíveis conseqüências na adesão ao tratamento. Ressalta-se a importância das recomendações sobre alimentação, contidas no Consenso Brasileiro 2 , sendo algumas específicas para o uso correto e absorção adequada de determinados medicamentos.

Os itens com maior facilidade de acerto foram dose, freqüência de administração e nome dos ARVs, resultado também coerente com os obtidos em outros trabalhos 6,9,10,19,29,30. Apesar da maior facilidade observada para esses itens, os resultados indicam falhas no conhecimento sobre a terapia ARV. A proporção de erros foi de $8,6 \%, 16,7 \%$ e $27,8 \%$ para, respectivamente, $d o$ se, freqüencia e nome. Este resultado preocupa, uma vez que esses itens são considerados de grande importância para o uso seguro dos medicamentos.

Uma propriedade útil da TRI é que, uma vez conhecida a compreensão $(\theta)$ de um indivíduo, é possível determinar a probabilidade de ele acertar um item. Exemplificando, um participante deste estudo com compreensão ( $\theta$ ) igual a 45,8 tem probabilidade de $62,9 \%, 91,6 \%, 71 \%, 58,1 \%$, $49 \%$ e $11,7 \%$ para acertar, respectivamente, nome, dose, freqüência de administração, alimentação, reações adversas e precauções dos medicamentos prescritos. Portanto, conhecendo-se o escore de um indivíduo pode-se prever quais itens ele terá mais chances de acertar ou errar, informando o domínio que o indivíduo possui do que foi avaliado. Este conhecimento das dificuldades e facilidades individuais de compreensão contribui para que os profissionais de saúde desenvolvam estratégias educacionais e habilidades ajustadas ao perfil do indivíduo, visando lidar com seu processo terapêutico complexo.

Esta estimação do nível de compreensão pode, inclusive, ser realizada na população estudada em momentos diferentes, para acompanhar a evolução desta medida ao longo do tempo. A informação obtida fornecerá subsídios para abordagens individualizadas de aconselhamento e monitoramento bem como para intervenções educacionais por equipes multidisciplinares, num esforço de ampliar a compreensão do paciente, e por conseqüência, melhorar sua adesão ao tratamento proposto.

Outra propriedade da TRI, sua maior vantagem, é permitir medidas que não dependam do grupo de indivíduos e nem do instrumento (teste), ou seja, são medidas com parâmetros invariantes. Essa propriedade pode ser de grande utilidade no planejamento e organização dos serviços de atenção ao portador de HIV. Ao proporcionar parâmetros invariantes, a TRI permite comparar indivíduos mesmo que eles tenham respondido a itens diferentes, em momentos diferentes. Como também permite comparar os resultados obtidos para populações diferentes, quando instrumentos com itens que meçam o mesmo traço latente são aplicados. Conhecendo-se os modelos estimados pela TRI e utilizando a equalização, é possível desenvolver estudos similares, em outros serviços de saúde na mesma região, ou em regiões diferentes. A equalização é uma técnica que equipara os parâmetros dos itens estimados em pesquisas distintas e níveis de compreensão de indivíduos de diferentes regiões, sobre uma mesma escala de compreensão, tornando os itens e os níveis de compreensão comparáveis. Assim é possível conhecer e avaliar diferentes situações, identificando necessidades e estratégias para uma melhor estruturação dos serviços e organização de seus processos de atenção.

Em relação ao método adotado na pesquisa, o modelo da TRI proposto mostrou-se um instrumento adequado para avaliar a compreensão da terapia ARV pelo paciente. Sumariamente, podese dizer que os escores elaborados para o construto compreensão, as interpretações dos parâmetros e os resultados obtidos pela aplicação dessa técnica estão de acordo com a expectativa da abordagem teórica do modelo. Entretanto, há necessidade de outros estudos testando a exclusão de alguns itens e a inclusão de outros novos itens visando obter um questionário com níveis mais elevados de qualidade, que se configure como um instrumento adequado de aferição do nível de compreensão de informações sobre medicamentos de uso crônico pelos pacientes, principalmente aqueles em uso de ARVs. Em particular, é oportuno desenvolver instrumento com maior capacidade de discriminar pacientes com níveis de compreensão acima da média. A TRI constitui-se em uma ferramenta eficaz que pode contribuir significativamente em muitas áreas do conhecimento, em especial em avaliações da compreensão da terapia prescrita, conforme demonstrado no presente trabalho. 
Os resultados observados neste estudo corroboram a importância de prover as informações necessárias, de assegurar que os pacientes compreendam o tratamento prescrito para o seu adequado cumprimento, especialmente no início da terapia ARV. A baixa adesão aumenta os riscos de falha terapêutica e da progressão da doença, que resultam em um impacto negativo sobre o custoefetividade da terapia ARV e a sustentabilidade dos benefícios do tratamento ao longo prazo ${ }^{31}$.
Neste sentido, é oportuno e necessário aprofundar estudos e identificar fatores que influenciam o nível de compreensão do tratamento ARV. O conhecimento desses fatores proporcionará subsídios aos enfoques educacionais que buscam apoiar o paciente em seu processo terapêutico e fortalecer sua autonomia. Contribuirá ainda para melhor entender e intervir efetivamente, de uma forma precoce, no processo de adesão ao tratamento pelo paciente.

\section{Resumo}

O objetivo do estudo foi desenvolver um escore para determinar o nível de compreensão de informações sobre terapia anti-retroviral (TARV) em pacientes no início do tratamento. Estudo transversal baseado em entrevistas com pacientes infectados pelo HIV em serviços públicos de referência (Belo Horizonte, Minas Gerais, Brasil). O escore da compreensão dos medicamentos foi obtido utilizando-se modelo de traço latente estimado pela Teoria de Resposta ao Item, após análise de concordância entre a resposta do paciente e a informação contida na prescrição. Realizou-se análise de regressão linear hierárquica para obter a compreensão global dos medicamentos, considerando cada classe de medicamentos (nível 1) e o individuo (nível 2). Dos 406 pacientes avaliados, 37,9\% não atingiram um nível mínimo de compreensão do tratamento. $O$ item com maior nível de dificuldade foi "precaução de uso". O item "dose" foi o que mais diferenciou os pacientes quanto à compreensão da TARV. Observouse alta proporção de pacientes com nível mínimo de compreensão da TARV, o que pode indicar um alto risco potencial de não-adesão à terapia. É necessário identificar fatores associados com este baixo nível de compreensão.

HIV; Anti-Retrovirais; Medicamentos com Prescrição

\section{Colaboradores}

M. G. B. Ceccato, F. A. Acurcio, P. F. Bonolo e M. D. C. Guimarães contribuíram no desenho do estudo, análise e interpretação de dados, elaboração e revisão crítica do texto e aprovação da versão final do artigo. C. C. César contribuiu na análise e interpretação de dados, elaboração e revisão crítica do texto e aprovação da versão final do artigo.

\section{Agradecimentos}

A pesquisa teve o apoio financeiro da Organização PanAmericana da Saúde/Organização Mundial da Saúde e Programa Nacional de DST e AIDS do Ministério da Saúde, além do apoio logístico da Coordenação Estadual de DST e AIDS da Secretaria Estadual de Saúde de Minas Gerais, do Núcleo de Educação em Saúde Coletiva da Universidade Federal de Minas Gerais (UFMG), do Grupo de Pesquisas em Epidemiologia e Avaliação em Saúde da UFMG e dos Departamentos de Medicina Preventiva e Social e de Farmácia Social da UFMG. 


\section{Referências}

1. Paterson DL, Swindells S, Mohr J, Brester M, Vergis EN, Squier C, et al. Adherence to protease inhibitor therapy and outcomes in patients with HIV infection. Ann Intern Med 2000; 133 Suppl 1:21-30.

2. Coordenação Nacional de DST e AIDS. Recomendações para a terapia anti-retroviral em adultos e adolescentes infectados pelo HIV - 2004. Brasília: Coordenação Nacional de DST e AIDS, Ministério da Saúde; 2003.

3. Akici A, Kalaça S, Ugurlu MÜ, Toklu HZ, Iskender E, Oktay S. Patient knowledge about drugs prescribed at primary healthcare facilities. Pharmacoepi Drug Saf 2004; 13 Suppl 12:871-6.

4. Ascione FJ, Kirscht JP, Shimp LA. Na assessment of different components of patient medication knowledge. Med Care 1986; 24 Suppl 11:1018-27.

5. Azevedo NDA. Conhecimento do paciente hospitalizado sobre o uso do digital. Rev Baiana Enferm 1987; 3:95-111.

6. Silva T, Schenkel EP, Sotero SS. Nível de informação a respeito de medicamentos prescritos a pacientes ambulatoriais de hospital universitário. Cad Saúde Pública 2000; 16:449-55.

7. Díaz JL, Lázaro GA, Redondo-de-Pedro S, SotoGarcía M, López-de-Castro F, Rodríguez-Alcalá FJ. ¿Comprenden los pacientes el tratamiento antibiótico prescrito? Aten Prim 2001; 28 Suppl 6:386-90.

8. Stone VE, Hogan JW, Schuman P, Rompalo AM, Howard AA, Korkontzelou C, et al. Antiretroviral regimen complexity, self-reported adherence, and HIV patients' understanding of their regimens: survey of women in the her study. J Acquir Immune Defic Syndr 2001; 28:124-31.

9. Jaye C, Hope J, Martin IRM. What do general practice patients know about their prescription medications? N Z Med J 2002; 115:U183.

10. Miller LG, Liu H, Hays RD, Golin CE, Ye Z, Beck CK, et al. Knowledge of antiretroviral regimen dosing and adherence: a longitudinal study. Clin Infec Dis 2003; 36:514-8.

11. Bartholomew DJ, Steele F, Moustaki I, Galbraith JI. The analysis and interpretation of multivariate data for social scientists. Boca Raton: Chapman \& Hall; 2002.

12. Soares TM. Utilização da teoria da resposta ao item na produção de indicadores sócio-econômicos. Pesquisa Operacional 2005; 25:83-112.

13. Soares JA, Andrade RJ. Nível socioeconômico, qualidade e eqüidade das escolas de Belo Horizonte. Ensaio: Avaliação e Políticas Públicas em Educação 2006; 14:107-26.

14. Soares JA, Collares ACM. Recursos familiares e o desempenho cognitivo dos alunos e ensino básico brasileiro. Dados 2006; 49:615-50.

15. Valle RC. Teoria de resposta ao item [Dissertação de Mestrado]. São Paulo: Instituto de Matemática e Estatística, Universidade de São Paulo; 1999.

16. Bonolo PF, César CC, Acúrcio FA, Ceccato MGB, Pádua CAM, Álvares J, et al. Non-adherence among patients initiating antiretroviral therapy: a challenge for health professionals in Brazil. AIDS 2005; 19(4 Suppl):5-13.
17. Ceccato MGB, Acurcio FA, Bonolo PF, Rocha GM, Guimarães MD. Compreensão de informações relativas ao tratamento anti-retroviral entre indivíduos infectados pelo HIV. Cad Saúde Pública 2004; 20:1388-97.

18. Vendramini CMM, Silva MC, Canale M. Análise de itens de uma prova de raciocínio estatístico. Psicologia em Estudo 2004; 9:487-98.

19. Birnbaum A. Some latent trait models and their use in inferring an examinee's ability. In: Lord FM, Novicks MR, editors. Statistical theories of mental test scores. Reading: Addison-Wesley Publishing; 1968. p. 397-472.

20. Andrade DF, Tavares HR, Valle RC. Teoria de resposta ao item: conceitos e aplicações. São Paulo: Associação Brasileira de Estatística; 2000. http:// www.inf.ufsc.br/ dandrade/TRI/ (acessado em 10/Dez/2005).

21. Baker FB. The basics of item response theory. Boston: Lawrence Rudner; 2001.

22. Hambleton RK. Principles and selected applications of item response theory. In: Linn RL, editor. Educational measurement. Phoenix: American Council on Education/Oryx Press; 1993. p. 147-200.

23. Lord FM. Applications of item response theory to practical testing problems. Hillsdale: Lawrence Erlbaum Associates; 1980.

24. Embretson E, Reise SP. Item response theory for psychologists. New Jersey: Lawrence Erlbaum Associates; 2000.

25. Flores-Mendoza CE, Abad FJ, Lele AJ. Item analysis of human figure drawings test: application of IRT. Psic Teor Pesqui 2005; 21:243-54.

26. Rasbash J, Browne W, Healy M, Cameron B, Charlton C. MLwiN. London: Multilevel Models Project, Institute of Education, University of London; 2001.

27. Centers for Disease Control and Prevention. 1993 revised classification system for HIV infection and expanded surveillance case definitions for AIDS among adolescents and adults. MMWR Recomm Rep 1992; 41(RR-17):1-19.

28. Hambleton RK, Swaminathan H, Rogers HJ. Fundamentals of item response theory. Newbury Park: Sage Publications; 1991.

29. Kay EA, Bailie GR, Bernstein A. Patient knowledge of cardio-respiratory drugs. J Clin Pharm Ther 1988; 13(4 Suppl):263-8.

30 Makaryus NA, Friedman EA. Patient's understanding of their treatment plans and diagnosis at discharge. Mayo Clin Proc 2005; 80(8 Suppl):991-4.

31. Acurcio FA, Puig-Junoy J, Bonolo PF, Ceccato MGB, Guimarães MDC. Análisis coste-efectividad de la adhesión inicial a la terapia antirretroviral entre individuos infectados por el VIH en Belo Horizonte, Brasil. Rev Esp Salud Pública 2006; 80:41-54.

Recebido em 04/Jun/2007

Aprovado em 29/Nov/2007 